\title{
Anteroposterior Airway Diameter during Sedation for Brain MRI in Children with and without Developmental Disabilities
}

\author{
Nirupama Kannikeswaran ${ }^{1 *}$, Swati Mody², Xinguang Chen³, Marwan Zidan4, \\ Lalitha Sivaswamy 5 \\ ${ }^{1}$ Wayne State University School of Medicine, Carman and Ann Adams Department of Pediatrics, Division of \\ Emergency Medicine, Children's Hospital of Michigan, Detroit, MI, USA \\ ${ }^{2}$ Wayne State University School of Medicine, Carman and Ann Adams Department of Pediatrics, Division of \\ Pediatric Radiology, Children's Hospital of Michigan, Detroit, MI, USA \\ ${ }^{3}$ Wayne State University, Detroit, MI, USA \\ ${ }^{4}$ Wayne State University School of Medicine, Detroit, MI, USA \\ ${ }^{5}$ Wayne State University School of Medicine, Carman and Ann Adams Department of Pediatrics, Division of \\ Neurology, Children's Hospital of Michigan, Detroit, MI, USA \\ Email: ${ }^{*}$ kkannike@dmc.org
}

Received 16 June 2014; revised 18 July 2014; accepted 2 August 2014

Copyright (C) 2014 by authors and Scientific Research Publishing Inc.

This work is licensed under the Creative Commons Attribution International License (CC BY). http://creativecommons.org/licenses/by/4.0/

(c) (i) Open Access

\section{Abstract}

Objective: Our study objective was to compare the anteroposterior (AP) oropharyngeal airway diameter measured using static MRI images in children with and without developmental disabilities during sedation. Methods: We performed a secondary analysis on a prospective, observational age-matched, 1:2 case-control study of children aged 3 - 10 years who were sedated for brain MRI. AP airway diameters were determined at the level of soft palate (airway_SP) and tongue (airway_T) from midline sagittal images by a single blinded radiologist. Results: Airway diameter was evaluated in 134 controls (C) and 68 cases (D). There was no difference in the overall adverse events (D: $30 \%$ C: $32.9 \%$; $p=0.7$ ) as well as hypoxia (D: $10 \%$; $: 9.3 \%, p=0.9$ ). There was no significant difference in mean airway_T (D: $10.62 \pm 3.48$ vs. C: $10.38 \pm 3.47$; $p=0.64$ ) and airway_SP diameter ( $D: 4.59 \pm 2.36$ vs. C: $5.17 \pm 3.9$; $p=0.14$ ) between the two groups. There was no significant difference in airway_T and airway_SP diameters amongst those who experienced and those who did not experience hypoxia in cases. Amongst controls however, the airway_SP was smaller in those who experienced hypoxia compared to those who did not experience hypoxia. Conclusions: There was no difference in the AP airway_T and airway_SP diameter during sedation using static MRI images in children with and without developmental disabilities. Changes in airway_SP was associated with hypoxia amongst controls in our study cohort.

\footnotetext{
${ }^{*}$ Corresponding author.
}

How to cite this paper: Kannikeswaran, N., Mody, S., Chen, X.G., Zidan, M. and Sivaswamy, L. (2014) Anteroposterior Airway Diameter during Sedation for Brain MRI in Children with and without Developmental Disabilities. Open Journal of Anesthesiology, 4, 191-196. http://dx.doi.org/10.4236/ojanes.2014.48027 


\section{Keywords}

\section{Sedation, Magnetic Resonance Imaging, Airway Diameter, Developmental Disabilities, Children}

\section{Introduction}

Respiratory depression and airway obstruction are the most common complications related to procedural sedation in children [1]. We had previously shown that there was no difference in sedation medication requirements and overall adverse events including hypoxia related to sedation between children with and without developmental disabilities [2]. Elwood et al. [3] had shown that the anteroposterior airway diameter measured at the level of soft palate using static Magnetic Resonance Imaging (MRI) images was $40 \%$ less in children with developmental disabilities when compared to normal children during sedation. They had concluded that this could make children with developmental disabilities more prone for upper airway obstruction during sedation.

The primary objective of this study was to compare the anteroposterior oropharyngeal airway diameter during sedation for brain MRI in children with and without developmental disabilities using static MRI images. The secondary objectives was to evaluate if there was any association between anteroposterior airway diameter and hypoxia in the study cohort. We hypothesized that there would no difference in the anteroposterior airway diameters during sedation for brain MRI in children with and without developmental disabilities. We further hypothesized that a narrower anteroposterior airway diameter would be associated with hypoxia in the study cohort.

\section{Methods}

\subsection{Study Design}

The current study was a planned secondary analysis of children who were enrolled in a prospective age matched 1:2 case control study of sedation medication requirements and adverse events related to sedation in children with (cases) and without developmental disabilities (controls). The study protocol was approved by the Institutional Review Board.

\subsection{Setting}

The study was conducted at the radiology department of a free standing tertiary care children's hospital. There are two MRI scanners (1.5 Tesla and 3.0 Tesla) in the imaging department and approximately 2500 children are sedated by an institutional sedation team every year for their MRI.

\subsection{Study Participants}

Children aged 3 - 10 years of age who required intravenous sedation for an elective, outpatient brain MRI were included for participation in the study. In addition to those patients excluded from the primary study [2], we also excluded from this study those children whose imaging study could not be completed due to inadequate sedation or due to sedation related adverse events as well as those whose airway could not evaluated by the study radiologist due to lack of digitalized images or presence of braces.

\subsection{Study Procedures}

We have previously discussed the details of the study methods of the parent study [2]. The parent study was a prospective, observational, age-matched, 1:2 case (children with developmental disability) control (children without developmental disability) study of children (3 - 10 years), sedated for brain MRI using a standardized institutional sedation protocol at a tertiary care children's hospital. Developmental assessment was performed using The Vineland Adaptive Behavioral Score (VABS) and by a pediatric neurologist. Patient demographics, type and dose of sedation medications, depth of sedation and adverse events were collected. We had defined hypoxia as oxygen saturation $\leq 90 \%$ for $\geq 30 \%$ seconds and requiring airway maneuvers.

For this study, all MRI images were reviewed by a single board certified pediatric radiologist who was 
blinded to the patient's group assignment. A midline sagittal image was identified by the presence of the pituitary stalk, fourth ventricle, aqueduct and anterior recess of the third ventricle. The narrowest anteroposterior airway diameter was measured perpendicular to the long axis of the airway posterior to the tongue (Airway_T) and at the level of the soft palate (Airway_SP) as described by Elwood et al. [3] (Figure 1).

All of these airway diameter measurements were performed by the study radiologist. We did not measure the transverse airway diameter or cross sectional area of the airway.

\subsection{Statistical Analysis}

All data variables were systematically coded and entered into an excel database by a single trained research assistant. Descriptive statistics was used to describe the study groups. Means and standard deviations for normally distributed continuous variables, medians and range for skewed continuous variables, frequencies and percentages for categorical variables are presented. Two-sample t-test (or Mann-Whitney method, based on the assumption satisfaction) was used to compare the continuous variables between the two groups and Chi-square test was used to compare the categorical variables. As a routine, type I error was set at $\mathrm{p}<0.05$. The statistical analysis was performed using SAS version 9.23 (SAS Institute, Cary, NI).

\section{Results}

Seventy children were designated as cases (D: those with developmental disability) and 140 children (C: normal children) were designated as controls for the parent study. For this study, airway diameter could be assessed only in 68 cases and 134 controls due failure of completion of study (5C), lack of digitalized images (2D) and presence of braces which prevented assessment of the airway diameter (1C).

There was no significant difference in patient demographics, mean patient weight, Body Mass Index (BMI), type of sedative used or mean sedation medication dosage between the two groups (Table 1).

There was no difference in the overall adverse events (D: 30\%; C: 32.9\%; $\mathrm{p}=0.7$ ) as well as hypoxia (D: $10 \%$; $: 9.3 \%, p=0.9$ ). There was no significant difference in the mean airway_T diameter (D: $10.62 \pm 3.48$ vs. C: $10.38 \pm 3.47 ; \mathrm{p}=0.64$ ) and mean airway_SP (D: $4.59 \pm 2.36$ vs. C: $5.17 \pm 3.9 ; \mathrm{p}=0.14$ ) between the two groups.

Results from stratified comparative analysis showed that in cases, there was no significant difference in airway_T and airway_SP diameters amongst those who experienced and those who did not experience hypoxia. However, in the control group, airway_T was larger and airway_SP was smaller in those children who experienced hypoxia compared to those who did not experience hypoxia (Table 2).

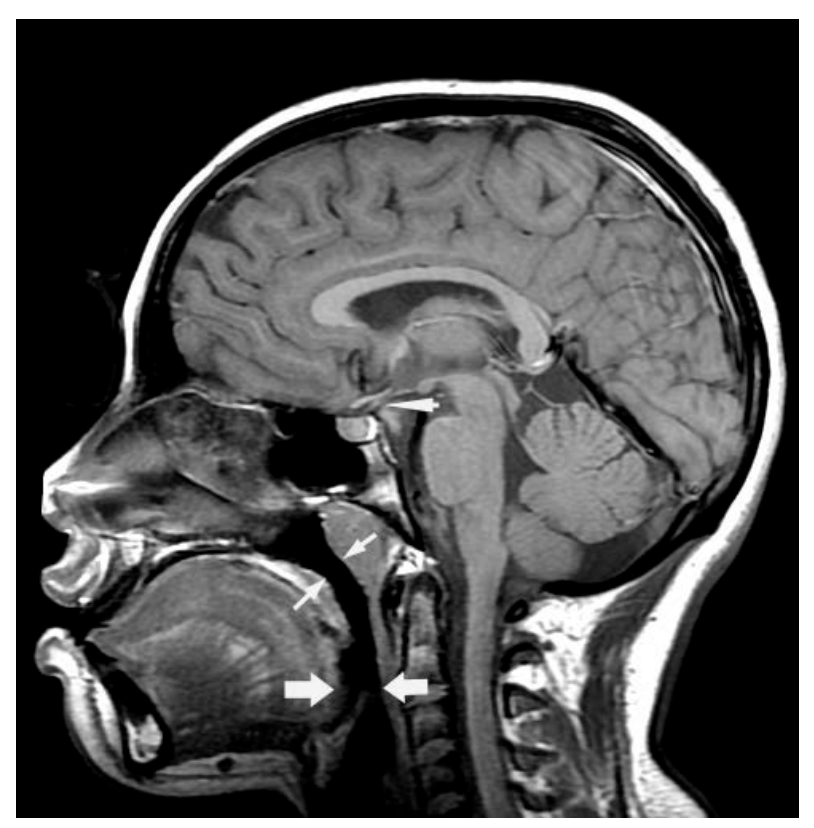

Figure 1. Arrowheads point to the pituitary stalk. 
Table 1. Patient demographics and sedation medications used: Children with and without developmental disabilities.

\begin{tabular}{|c|c|c|c|c|}
\hline \multicolumn{2}{|c|}{ Variable } & $\begin{array}{l}\text { Cases } \\
\text { (Children with developmental disability) } \\
\qquad \mathrm{n}=68\end{array}$ & $\begin{array}{l}\text { Controls (Children without } \\
\text { developmental disability) } \\
\text { n = } 134\end{array}$ & $\mathrm{p}$ \\
\hline \multirow{2}{*}{ Gender } & Male & 47 (69.1\%) & $79(58.9 \%)$ & \\
\hline & Female & $21(30.9 \%)$ & $55(41.0 \%)$ & \\
\hline \multirow{2}{*}{ ASA } & Class 1 & $18(26.5 \%)$ & $86(64.2 \%)$ & \\
\hline & Class 2 & $50(73.5 \%)$ & 48 (35.8\%) & \\
\hline \multicolumn{2}{|c|}{ Age (Median \pm IQR) } & $5.0 \pm 3.0$ & $5.0 \pm 3.0$ & 0.98 \\
\hline \multicolumn{2}{|c|}{ Weight (Median \pm IQR) } & $19.7 \pm 8.3$ & $21.6 \pm 8.1$ & 0.23 \\
\hline \multirow{2}{*}{\multicolumn{2}{|c|}{ BMI (Median \pm IQR) }} & $17.5 \pm 7.6$ & $16.6 \pm 3.9$ & 0.14 \\
\hline & & Nembutal: 68 (100\%) & Nembutal: 134 (100\%) & 0.15 \\
\hline \multirow{2}{*}{\multicolumn{2}{|c|}{ Type of Sedative Medication ${ }^{* *}$}} & Fentanyl: 37 (54.4) & Fentanyl: 79 (58.9) & 0.66 \\
\hline & & Midazolam: 33 (48.5) & Midazolam: 61 (45.5) & 0.59 \\
\hline \multicolumn{2}{|c|}{ Mean Nembutal Dosage in mg/kg (SD) } & $4.77 \pm 1.54$ & $4.67 \pm 1.69$ & 0.67 \\
\hline \multicolumn{2}{|c|}{ Mean Fentanyl Dosage in mcg/kg (SD) } & $1.15 \pm 0.42$ & $1.13 \pm 0.44$ & 0.86 \\
\hline \multicolumn{2}{|c|}{ Mean Midazolam Dosage in mg/kg (SD) } & $0.15 \pm 0.17$ & $0.11 \pm 0.14$ & 0.1 \\
\hline
\end{tabular}

${ }^{* *} 45 \%$ of study patients received a combination of nembutal and fentanyl and $33.6 \%$ of patients received a combination of nembutal and midazolam.

Table 2. Comparison of airway diameters with hypoxia in children stratified by developmental status.

\begin{tabular}{ccccc}
\hline & \multicolumn{2}{c}{ Cases (Children with developmental disability) } & \multicolumn{2}{c}{ Controls (Children without developmental disability) } \\
\cline { 2 - 5 } & Airway_T Mean (SD) & Airway_SP Mean (SD) & Airway_T Mean (SD) & Airway_SP Mean (SD) \\
\hline Hypoxia & $10.19(3.80)$ & $5.38(2.41)$ & $12.40(3.56)$ & $4.12(2.41)$ \\
No hypoxia & $10.73(3.43)$ & $4.39(2.32)$ & $9.91(3.29)$ & $5.41(3.06)$ \\
t value (p) & $0.51(0.613)$ & $1.42(0.162)$ & $3.36(0.001)$ & $1.97(0.05)$ \\
\hline
\end{tabular}

\section{Discussion}

Our study results showed that there was no difference in the anteroposterior airway diameters measured using static MRI images during sedation for brain MRI between children with and without developmental disabilities. These results are somewhat different from what was shown by Elwood et al. [3] who had demonstrated no difference in airway_T diameters but had shown a $40 \%$ difference in airway_SP between children with and without developmental disabilities. Although the reasons for this are not clear, it could be because of the fact that we excluded children with ASA classification $\geq 3$ which resulted in selection of children with mild developmental delay in our study. In addition, Elwood et al. also excluded those children who received midazolam for sedation for its presumed effect of decreasing airway muscle tone. In our study, around $45 \%$ of cases and controls received midazolam which should have affected the airway diameter in both the groups. Furthermore, we did not match the cases and controls for the sedative type or dose. Given that different sedatives could have varying effects on airway muscle tone and diameter, this could explain the lack of difference in the airway diameters amongst our two study groups.

A recent study of the morphologic changes in upper airway diameter during propofol sedation demonstrated significant differences in the airway diameter between sedated and awake states [4]. During sedation, the upper airway was oblong shaped, with the anteroposterior diameter being larger than the transverse diameter. During awakening on the other hand, the shape of the upper airway changed such that the transverse diameter was larger. There was no change in the cross sectional area of the upper airway between sedated and awake states. It is possible that we would have shown a difference in airway diameters between our study groups had we measured the transverse airway diameters. 
During sedation, it has been shown that neuromuscular control of the upper airway muscles through glossopharyngeal and vagus nerves is inhibited to a greater degree than diaphragmatic activity (phrenic nerve). This differential inhibition of the hypoglossal and phrenic nerve has been demonstrated with halothane, thiopental and diazepam [5]. The negative inspiratory pressures that develop with diaphragmatic contraction cause a reduction in the pharyngeal diameter leading to a narrowing of the anteroposterior distance between the posterior pharynx and the soft palate, epiglottis and, to a lesser degree, the base of the tongue. While it was previously thought that the base of the tongue was the primary cause of upper airway obstruction during sedation, several studies of the airway in sedated children and adults have demonstrated that the soft palate and epiglottis are the most likely structures causing airway obstruction [6]-[9]. This was shown in our study by a narrower airway_SP being associated with hypoxia although why this effect was only seen in normal children and not those with developmental disabilities is not known. In our previous study [2] comparing sedation related adverse events in children with and without developmental disabilities, upper airway obstruction was more commonly seen in normal children (17.9\% in normal children vs. $12.9 \%$ in those with developmental disabilities) whereas apnea was more common in children with developmental disabilities ( $4.3 \%$ in cases versus $0 \%$ in controls) leading us to postulate that the mechanism of sedation related hypoxia was different in these two groups of children.

\section{Limitations}

We evaluated airway diameters using static MRI images and did not perform a dynamic assessment. We studied only the anteroposterior airway diameter and did not study the transverse diameter or the cross sectional area of the airway. The study results could have been different had we measured transverse airway diameter as well as used dynamic measurements of airway diameter. The airway diameter measurements could have been influenced by the head position and extension during MRI, the phase of respiration as well as airway maneuvers performed to overcome airway adverse events. Motion artifacts during the imaging study could have influenced the airway diameter measurements. However, all of the study subjects were sedated to a Ramsey Sedation Score of $>4$ minimizing motion during imaging. We did not match the cases and controls for the sedative type or dose. We did not study children with a specific etiology of developmental disability or correlate airway diameters with the degree of developmental disability. The airway diameter measurements were performed by a single blinded radiologist which could have introduced inter observer variability bias. Also, even though the radiologist was blinded to the patient's group assignment, it is possible that they were aware of the patient's developmental status based on the interpretation of the MRI images. The airway diameter measurements could have been influenced by the specific sedatives used at our institution and hence the results may not be applicable to other centers which use a different sedation protocol.

\section{Conclusion}

In our study cohort, there was no difference in the anteroposterior airway_T and airway_SP diameters using static MRI images between children with and without developmental disabilities during sedation for brain MRI. Decrease in Airway_SP was associated with hypoxia in children without developmental disabilities.

\section{Funding and Financial Assistance}

The parent study was funded through the investigator initiated research program of Blue Cross Blue Shield Foundation of Michigan. This study was a planned secondary analysis of the initial study. There was no funding for this secondary study.

The sponsor had no involvement in: 1) study design; 2) the collection, analysis, and interpretation of data; 3) the writing of the manuscript; and 4) the decision to submit the manuscript for publication.

\section{References}

[1] Cravero, J.P., Blike, G.T., Beach, M., Gallagher, S.M., Hertzog, J.H., Havidich, J.E., et al. (2006) Incidence and Nature of Adverse Events during Pediatric Sedation/Anesthesia for Procedures outside the Operating Room: A Report from the Pediatric Sedation Research Consortium. Pediatrics, 118, 187-196. http://dx.doi.org/10.1542/peds.2006-0313

[2] Kannikeswaran, N., Sethuraman, U., Sivaswamy, L., Chen, X. and Mahajan, P.V. (2012) Children with and without Developmental Disabilities: Sedation Medication Requirements and Adverse Events Related to Sedation. Pediatric 
Emergency Care, 28, 1036-1040. http://dx.doi.org/10.1097/PEC.0b013e31826cad7e

[3] Elwood, T., Hansen, L.D. and Seeley, J.M. (2001) Oropharyngeal Airway Diameter during Sedation in Children with and without Developmental Delay. Journal of Clinical Anesthesia, 13, 482-485. http://dx.doi.org/10.1016/S0952-8180(01)00312-9

[4] Litman, R.S., Weissend, E.E., Shrier, D.A. and Ward, D.S. (2002) Morphologic Changes in the Upper Airway of Children during Awakening from Propofol Sedation. Anesthesiology, 96, 607-611. http://dx.doi.org/10.1097/00000542-200203000-00016

[5] Nishino, T., Shirahata, M., Yonezawa, T. and Honda, Y. (1984) Comparison of Changes in the Hypoglossal and the Phrenic Nerve Activity in Response to Increasing Depth of Anesthesia in Cats. Anesthesiology, 60, 19-24. http://dx.doi.org/10.1097/00000542-198401000-00005

[6] Boidin, M.P. (1985) Airway Patency in the Unconscious Patient. British Journal of Anaesthesia, 57, 306-310. http://dx.doi.org/10.1093/bja/57.3.306

[7] Abernethy, L.J., Allan, P.L. and Drummond, G.B. (1990) Ultrasound Assessment of the Position of the Tongue during Induction of Anaesthesia. British Journal of Anaesthesia, 65, 744-748. http://dx.doi.org/10.1093/bja/65.6.744

[8] Nandi, P.R., Charlesworth, C.H., Taylor, S.J., Nunn, J.F. and Dore, C.J. (1991) Effect of General Anesthesia on the Pharynx. British Journal of Anaesthesia, 66, 157-162. http://dx.doi.org/10.1093/bja/65.6.744

[9] Mathru, M., Esch, O., Lang, J., Herbert, M.E., Chalijub, G., Goodacre, B., et al. (1996) Magnetic Resonance Imaging of the Upper Airway. Anesthesiology, 84, 273-279. http://dx.doi.org/10.1097/00000542-199602000-00004 
Scientific Research Publishing (SCIRP) is one of the largest Open Access journal publishers. It is currently publishing more than 200 open access, online, peer-reviewed journals covering a wide range of academic disciplines. SCIRP serves the worldwide academic communities and contributes to the progress and application of science with its publication.

Other selected journals from SCIRP are listed as below. Submit your manuscript to us via either submit@scirp.org or Online Submission Portal.
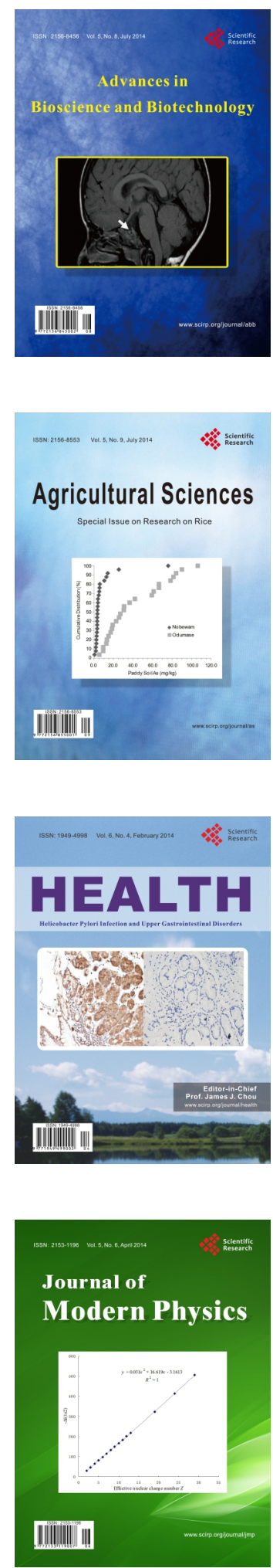
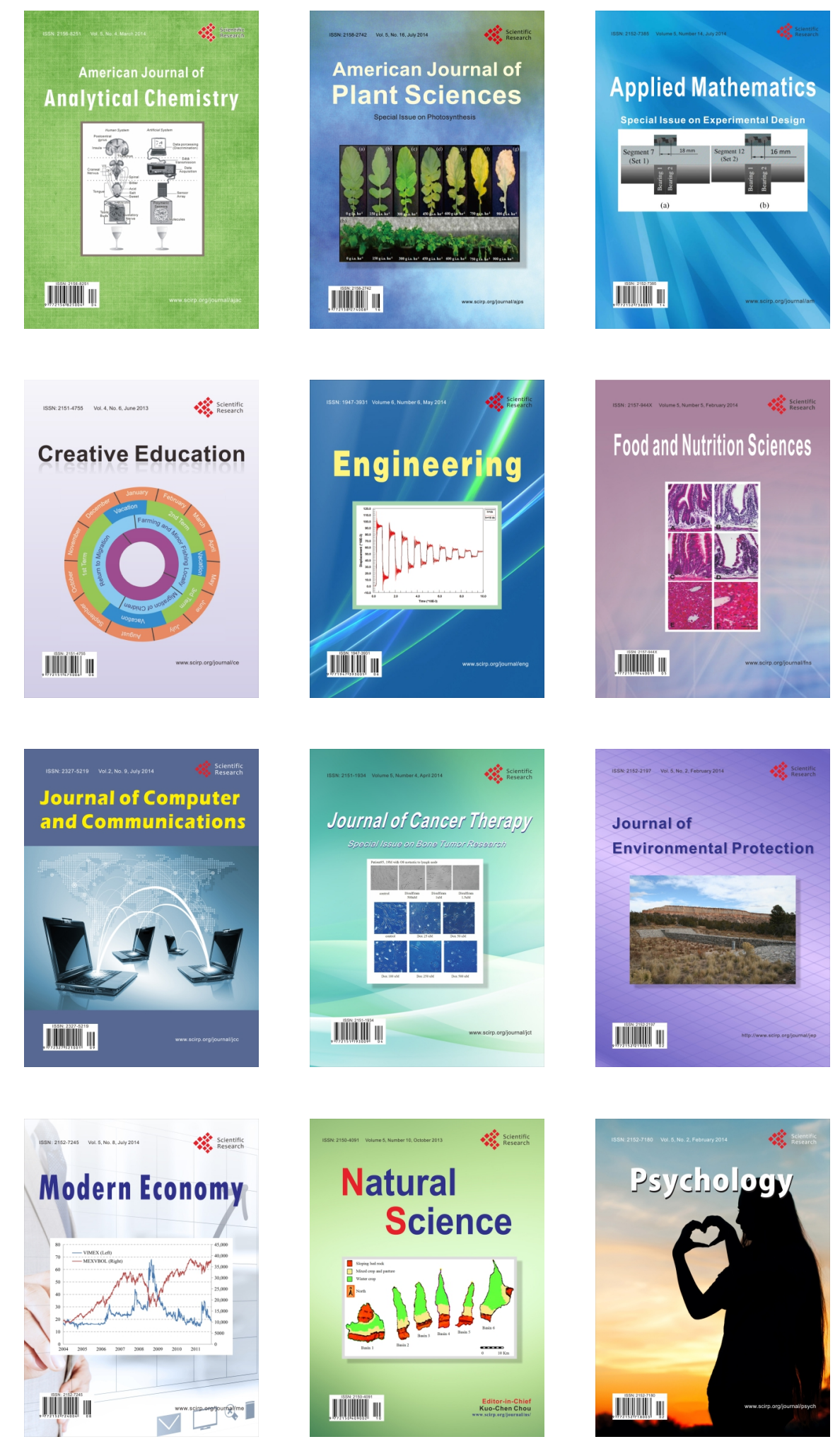